\title{
Computational Fluid Dynamics Simulations of Chimpanzee Nasal Airflow for Different Respiratory Modes
}

\section{Kaouthar Samarat $^{{ }^{*}}$, Sho Hanida ${ }^{2}$, Shigeru Ishikawa ${ }^{3}$, Kazunori Kotani $^{4}$, Teruo Matsuzawa ${ }^{4}$}

${ }^{1}$ Ishikawa Resin Industry Co., Ltd., Ta 1-8 Utani, Kaga-shi, Ishikawa 922-0312, Japan; ${ }^{2 K a n a z a w a}$ Institute of Technology, 7-1 Ohgigaoka, Nonoichi-shi, Ishikawa 921- 8501, Japan; ${ }^{3}$ Kanazawa Municipal Hospital, 3-7-3 Heiwamachi, Kanazawa-shi, Ishikawa 921-8105, Japan; ${ }^{4} J a p a n$ Advanced Institute of Science and Technology, 1-1 Asahidai, Nomi-shi, Ishikawa 923-1292, Japan

\section{ABSTRACT}

In this study, we investigated the airflow and air-conditioning within an anatomically accurate computer model of the nasal airways belonging to a healthy adult chimpanzee. The finite volumes method was used to compute unsteady turbulent flows with physiological flow rates of $2.2 \sin (2 \pi \mathrm{t} / 3.4)[\mathrm{m} / \mathrm{s}], 1.8 \sin$ $(2 \pi \mathrm{t} / 1.6)[\mathrm{m} / \mathrm{s}]$, and $4.4 \mathrm{sin}(2 \pi \mathrm{t} / 1.4)[\mathrm{m} / \mathrm{s}]$, mimicking breathing at rest state, shallow breathing under light stress, and a sniffing phase, respectively. Turbulent k-omega model was used to simulate unsteady respiratory phases whereas the turbulent k-epsilon model was used to simulate the sniffing phase. Simulation results argued that assuming a steady laminar inhalation state to investigate the air-conditioning performance within chimpanzee nasal cavity may be exaggerated. The outcomes of this study might potentially contribute in accumulating standardized biological information on healthy chimpanzee, and so increasing the ability to care for it as an endangered species.

Keyword: Chimpanzee nasal computer model, Chimpanzee nasal airflow, Computational fluid dynamics, Different breathing modes, Nasal air-conditioning, Turbulent unsteady flow.
*Correspondence to Author:

Kaouthar Samarat

Ishikawa Resin Industry Co., Ltd., Ta 1-8 Utani, Kaga-shi, Ishikawa 922-0312, Japan

Email: s1521006@jaist.ac.jp

How to cite this article:

Kaouthar Samarat, Sho Hanida, Shigeru Ishikawa, Kazunori Kotani, Teruo Matsuzawa. Computational Fluid Dynamics Simulations of Chimpanzee Nasal Airflow for Different Respiratory Modes. International Journal of Bioscience and Medicine, 2018; 2:8.

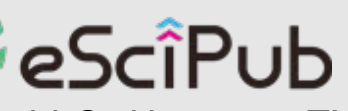

eSciPub LLC, Houston, TX USA. Website: http://escipub.com/ 


\section{Introduction}

In mammals, the respiratory air flows from the external nostrils through the nasal passage into the pharyngeal cavity [1], [2]. Mammalian nasal fossa contains interconnected airways network wherein inhaled air-conditioning in terms of temperature and humidity [3], olfactory sensing, and contaminants filtering occur [1], [2]. Respiratory air exchanges heat and moisture with nasal mucosal tissue to meet the conditions required in the lungs (fully saturated at body temperature).

Because of its complex topology, the nasal architecture has been far less explored in most mammals leaving comparison context vague. The advancement in medical imaging and computational power have significantly improved the time required to reconstruct threedimensional (3D) anatomical models and determine airflow patterns. Consequently, it is now possible to generate nasal geometries from several animals which may be used for interspecies comparisons of nasal airways variability to understand the functional implications regarding respiratory and olfactory airflow [4]. So far, studies of nasal anatomy in mammalian animals included lagomorphs [5], rodents [6], carnivores [7], [8], and ungulates [9], [10].

Recently, there has been an increase in the number of computational fluid dynamics (CFD) simulations involving realistic nasal cavities of Old World monkeys [11], [12] and chimpanzee [12]. [11] investigated the contribution of the maxillary sinus to air-conditioning performance, using CFD simulations based on 3D topological computer models, derived from computed tomography (CT) scans, of the nasal cavity of four Japanese macaques, two rhesus macaques, and a savanna monkey. [12] used a CFD model to compare nasal air-conditioning principles and performance in humans, chimpanzees, and macaques; based on digital topological models of the nasal passage reconstructed from medical imaging of six human volunteers, four chimpanzees, four
Japanese macaques, and two rhesus macaques.

Even though [12] carefully evaluated nasal airconditioning performance among the three genera (human, chimpanzee, and Old-World monkeys) by simulating varied ambient atmospheric conditions, they have only reported constant respiratory flow models which may not represent what actually occurs during unstable tidal airflow. Furthermore, they assumed that nasal airflow is laminar whereas turbulent models have been used for airflow simulations in human nasal cavities [13], [14].

In this study, we investigated the airflow and airconditioning within an anatomically accurate computer model [15] of the nasal airways belonging to a healthy adult chimpanzee [16]. The finite volumes method was used to compute unsteady turbulent cases with physiological flow rates of $2.2 \sin (2 \pi \mathrm{t} / 3.4)[\mathrm{m} / \mathrm{s}], 1.8 \sin (2 \pi \mathrm{t} / 1.6)$ $[\mathrm{m} / \mathrm{s}]$, and $4.4 \sin (2 \pi \mathrm{t} / 1.4)[\mathrm{m} / \mathrm{s}]$, mimicking breathing at rest state, shallow breathing under light stress state, and a sniffing phase, respectively. Whereas similar CFD analysis performed in the same subject compared the air-conditioning performance of human, chimp, and macaque [12], here we report realistic computations using a CFD model reliable for small-bodied chimpanzee which has a thinner mucosal layer than human. Studying a range of chimp specimens is presently considered beyond the scope of this paper.

The results of this study might potentially contribute in accumulating standardized biological information on healthy chimpanzee, and so increasing the ability to care for it as an endangered species.

\section{Materials and Methods}

\subsection{Ethics statement}

The scanning experiment was carried out in accordance with the third edition of the Guidelines for the Care and Use of laboratory Primates by the Primate Research Institute, Kyoto University (KUPRI), Inuyama, Japan. The experimental protocol was approved by the 
Animal Welfare and Care Committee of the same institute (Permit Number: 2012-075). The chimpanzee, Pan troglodytes, which is reared at the same institute, was scanned with the computer topography scanner (Ateion Premium4, Toshiba Medical Systems Co.) at KUPRI. The animal was anesthetized intramuscularly with $3.5 \mathrm{mg}$ ketamine hydrochloride (Sankyo-Parkle-Davis \& Co., Inc.) and $0.035 \mathrm{mg}$ medetomidine hydrochloride (Meiji Seika Kaisha Ltd.) per kilogram of body weight. This procedure was conducted after it was sedated orally using $3.75 \mathrm{mg}$ of droperidol in $1.5 \mathrm{ml}$ of orange juice, and the anesthesia was maintained with isoflurane (Dainippon Sumitomo Pharma Co., Ltd.) delivered in oxygen through a precision vaporizer and a re-breathing circuit.

\subsection{Anatomical data}

The CT scanning used in this study consisted of 401 slices, spanned from mouth tip to just anterior to larynx, of the head of an adult chimpanzee (estimated age: 35-year-old; weight: $44.1 \mathrm{~kg}$ ), Pan troglodytes reared at KUPRI [16]. Each slice was composed of 512 x 512 pixels in the DICOM format. Scans resolution was $0.351 \mathrm{~mm} / \mathrm{pixel}$ and scans pitch was $0.5 \mathrm{~mm} / \mathrm{slice}$. The scans were performed in three different axial positions. The CT scans are available on the digital morphology museum (DMM) database (http://dmm.pri.kyoto-u.ac.jp), of the Primate Research Institute, Kyoto University, Inuyama, Japan (CT scans id: PRICT \#467 and 468).

\subsection{Reconstruction of nasal topology}

Black areas of CT scans represented nasal airways. They were extracted, based on a threshold of brightness using Avizo 7 (FEI Visualization Sciences Group, Hillsboro, $\mathrm{OH}$, USA), thus voxel data of the nasal cavity were reconstructed. After converting the voxel data into STereoLithography (STL) data, they were modified to derive a 3D smooth surface using Magics 9.5 (Materialize Inc., Leuven, Belgium). Finally, an unstructured tetrahedron mesh was generated using Gambit 2.4 (ANSYS Inc.,
Canonsburg, PA, USA). The computational mesh contained about $3.7 \times 10^{6}$ tetrahedron cells, $7.6 \times 10^{6}$ triangles, and $7.4 \times 10^{5}$ nonduplicated nodes. Its surface area and total volume were $185.41 \mathrm{~cm}^{2}$ and $41.64 \mathrm{~cm}^{3}$, respectively [15].

\subsection{Computational model of the nasal airflow}

We used an air flow model with heat and humidity transport [17], adapted to reflect chimpanzee respiratory physiology. The governing equations were resolved with the aid of FLUENT (ANSYS Inc., Canonsburg, PA, USA) using the finite volumes scheme.

\subsection{Boundary conditions}

Boundary conditions used to obtain the results in this paper were implemented in a manner similar to that stated by [18]. Physiologically realistic estimates of respiratory rate and tidal volume were calculated based on the body weight of the subject as reported by [19] and [20].

\subsection{Computational parameters}

Simulations of airflow inside the chimpanzee nasal airways were performed with the aid of FLUENT (ANSYS Inc., Canonsburg, PA, USA). Numerical computations were performed with implicit time stepping and a pressure-based solver. Pressure-velocity coupling was accomplished with SIMPLE approach. GreenGauss Cell Based gradient option was used to determine spatial gradient. Second Order and Quick discretization schemes were used for pressure and other remaining variables respectively. Turbulent k-omega model was used to simulate unsteady respiratory states whereas the turbulent k-epsilon model was used to simulate the sniffing phase. Computation was run on Cray XC30. Parallel computation was performed on 18 processors using the parallelization tool of FLUENT. A norm less than $10^{-6}$ was set as convergence criterion to terminate the iterations of energy; other norms were less than $10^{-5}$ except for the water mass fraction which was set to less than $10^{-3}$. The under-relaxation factors were kept as default. 
Graphical results were displayed using Avizo 7.0.

\section{Results and Discussion}

We investigated turbulent unsteady states to analyze the airflow patterns within a realistic chimpanzee computer nasal model. The CFD simulations were performed for three different breathing modes: Breathing at rest state, shallow breathing under light stress, and a sniffing phase. The following figure describes the configuration of each case.

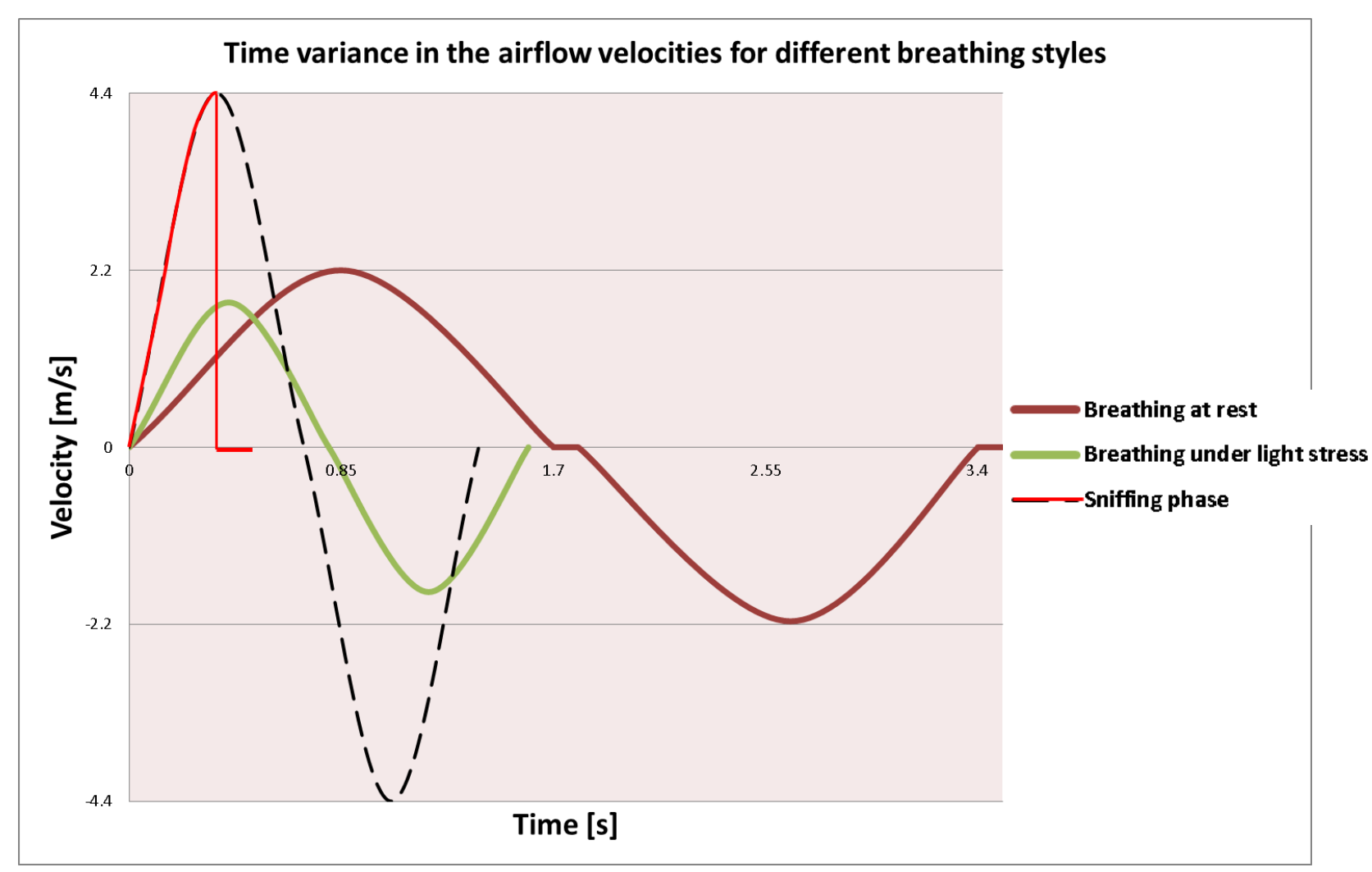

Figure 1. Time variance in the airflow velocities for different breathing modes: Sniffing phase (red); Shallow breathing under light stress (green); and breathing at rest state (brown). The horizontal axis represents the time [s] and the vertical axis represents the nasal airflow velocity [m/s].

\subsection{Breathing at rest state}

Time variance of the airflow velocity for breathing at rest state is defined by a sine wave function as $V=2.2 \sin (2 \pi t / 3.4) \quad[\mathrm{m} / \mathrm{s}]$. Inspiratory phase, $1.7 \mathrm{~s}$; Resting phase, $0.1 \mathrm{~s}$; Expiratory phase, $1.7 \mathrm{~s}$; Resting phase, $0.1 \mathrm{~s}$. We assumed a tidal volume TV $=395 \mathrm{ml}$ with 18 breaths $/ \mathrm{min}$.

\subsection{Shallow breathing under light stress}

Time variance of the airflow velocity for shallow breathing under light stress is defined by a sine wave function as $V=1.8 \sin (2 \pi \mathrm{t} / 1.6)[\mathrm{m} / \mathrm{s}]$. Inspiratory phase, $0.8 \mathrm{~s}$; Expiratory phase, $0.8 \mathrm{~s}$. We assumed a tidal volume $\mathrm{TV}=200 \mathrm{ml}$ with 37 breaths $/ \mathrm{min}$.

\subsection{Sniffing}

Time variance of the airflow velocity for sniffing phase is defined by a sine wave function as $\mathrm{V}=$ $4.4 \sin (2 \pi \mathrm{t} / 1.4)[\mathrm{m} / \mathrm{s}]$. Sniffing phase, $0.35 \mathrm{~s}$; Resting phase, $0.1 \mathrm{~s}$. We assumed a tidal volume $\mathrm{TV}=200 \mathrm{ml}$. 


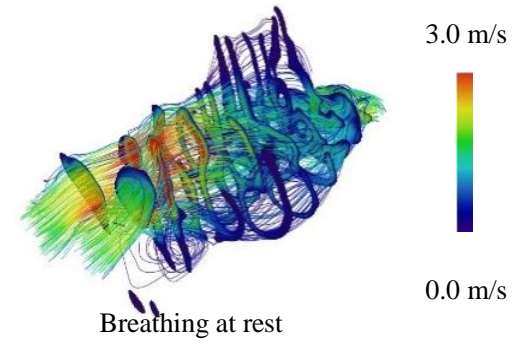

Inspiratory phase $(1 / 4$ cycle $), V \max =4.0 \mathrm{~m} / \mathrm{s}$

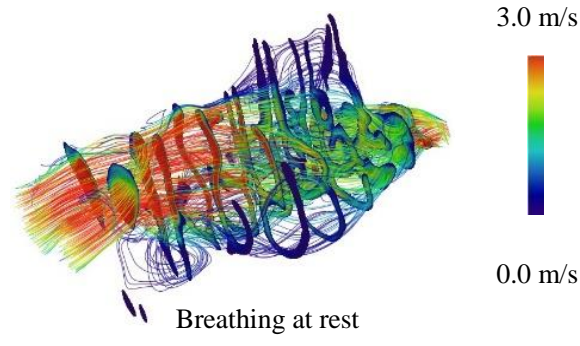

Inspiratory phase $(1 / 2$ cycle $), V \max =5.8 \mathrm{~m} / \mathrm{s}$

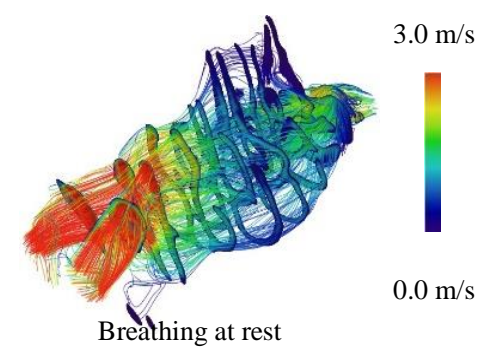

Expiratory phase (1/4 cycle), $\mathrm{V} \max =4.6 \mathrm{~m} / \mathrm{s}$

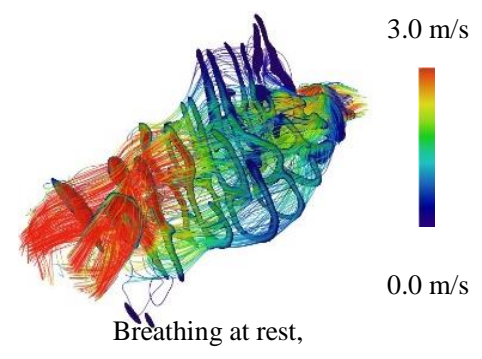

Expiratory phase $(1 / 2$ cycle $), \operatorname{Vmax}=6.1 \mathrm{~m} / \mathrm{s}$

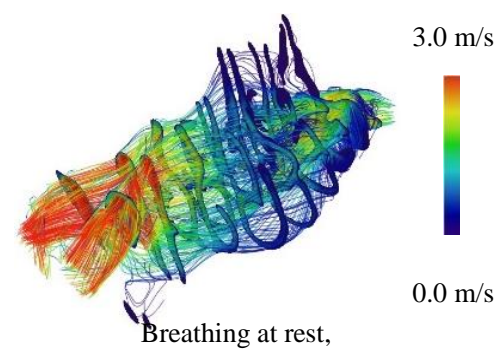

Expiratory phase (3/4 cycle), $\mathrm{Vmax}=4.3 \mathrm{~m} / \mathrm{s}$

Figure 2. Airflow pattern and velocity distribution within chimpanzee nasal airways during breathing at rest state: The streamlines and the contour planes indicate air streams and structure of flow velocity, respectively.

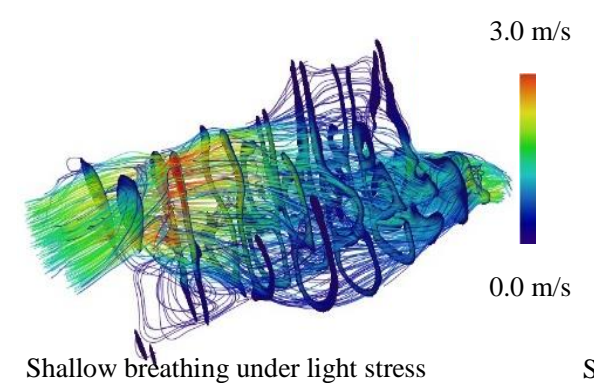

Shallow breathing under light stress

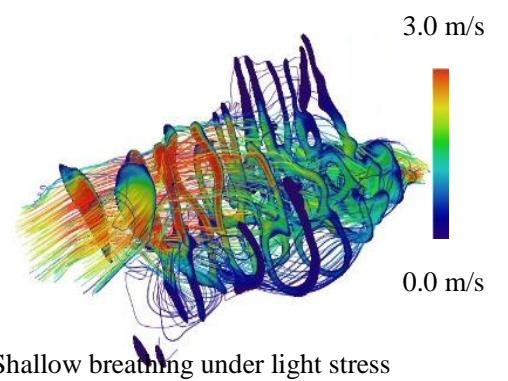

Inspiratory phase $(1 / 2$ cycle $), \mathrm{V} \max =5.0 \mathrm{~m} / \mathrm{s}$

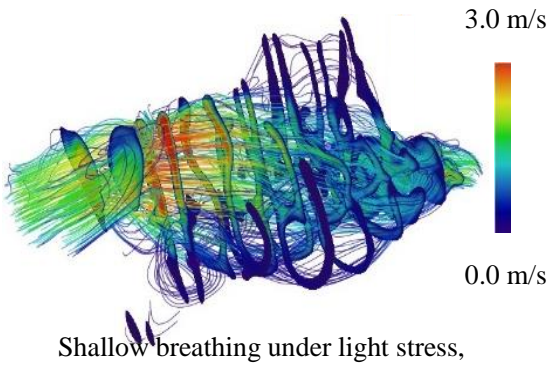

Inspiratory phase $(3 / 4$ cycle $), V \max =3.5 \mathrm{~m} / \mathrm{s}$

$3.0 \mathrm{~m} / \mathrm{s}$

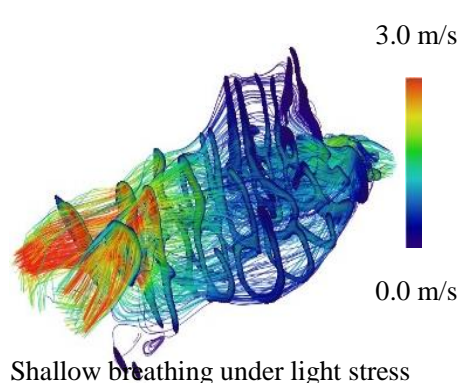

Shallow bttathing under light stress

Expiratory phase (1/4 cycle), Vmax $=3.65 \mathrm{~m} / \mathrm{s}$

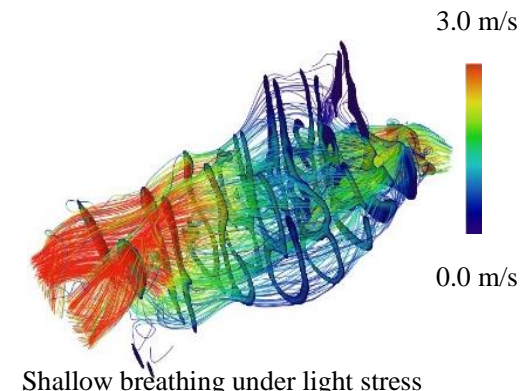

Shallow breathing under light stress

Expiratory phase $(1 / 2$ cycle $), \mathrm{Vmax}=5.1 \mathrm{~m} / \mathrm{s}$

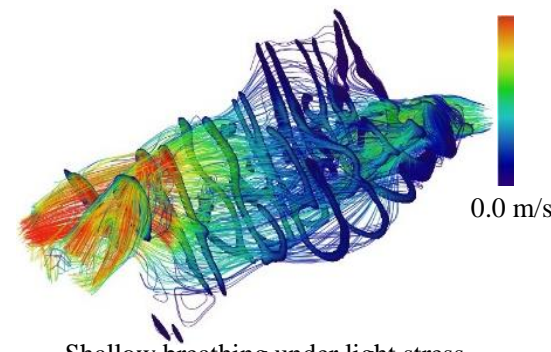

Shallow breathing under light stress,

Expiratory phase ( $3 / 4$ cycle), $V \max =3.7 \mathrm{~m} / \mathrm{s}$

Figure 3. Airflow pattern and velocity distribution within chimpanzee nasal airways during shallow breathing under light stress: The streamlines and the contour planes indicate air streams and structure of flow velocity, respectively. 


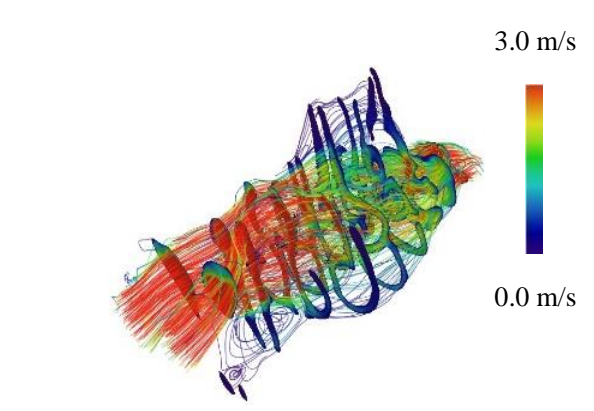

Sniffing phase ( $1 / 2$ cycle), $V \max =7.6 \mathrm{~m} / \mathrm{s}$

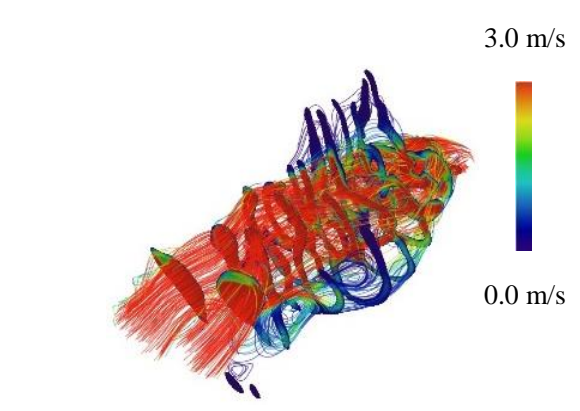

Sniffing phase ( $1 / 1$ cycle), Vmax $=11.6 \mathrm{~m} / \mathrm{s}$

Figure 4. Airflow pattern and velocity distribution within chimpanzee nasal airways during sniffing phase: The streamlines and the contour planes indicate air streams and structure of flow velocity, respectively.

Figure 5 shows the simulated temperature distribution within chimpanzee nasal cavity. The horizontal axis represents the contour plane number starting from nostril tips to nasopharyngeal region, the vertical axis represents temperature for different breathing modes including the laminar steady inhalation phase [12]. Although roughly all cases exhibited the same tendency of temperature distribution, the inhaled air wasn't adjusted similarly before reaching the pharyngeal region (contour plane number 9).

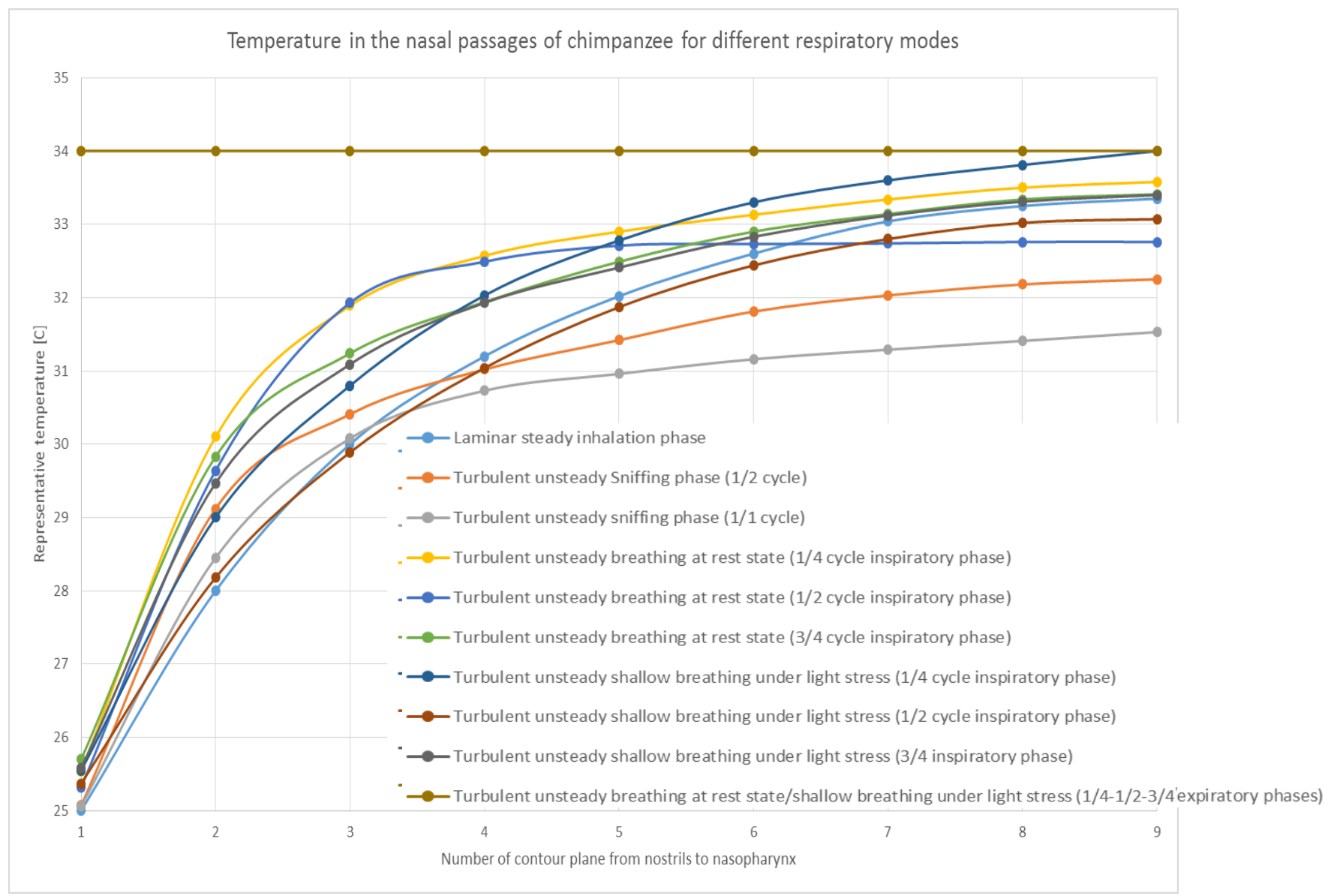

Figure 5. Temperature distribution within chimpanzee nasal cavity for different breathing modes: Horizontal axis represents number of contour plane from nostrils to pharynx. Vertical axis represents temperature $\left[{ }^{\circ} \mathrm{C}\right]$. 
Figure 6 shows the simulated water mass fraction distribution within chimpanzee nasal cavity. The horizontal axis represents the contour plane number starting from nostril tips to nasopharyngeal region, the vertical axis represents water mass fraction for different breathing modes including the laminar steady inhalation phase [12]. Although roughly all cases exhibited the same tendency of water mass fraction distribution, the inhaled air wasn't moisturized similarly before reaching the pharyngeal region (contour plane number 9 ).

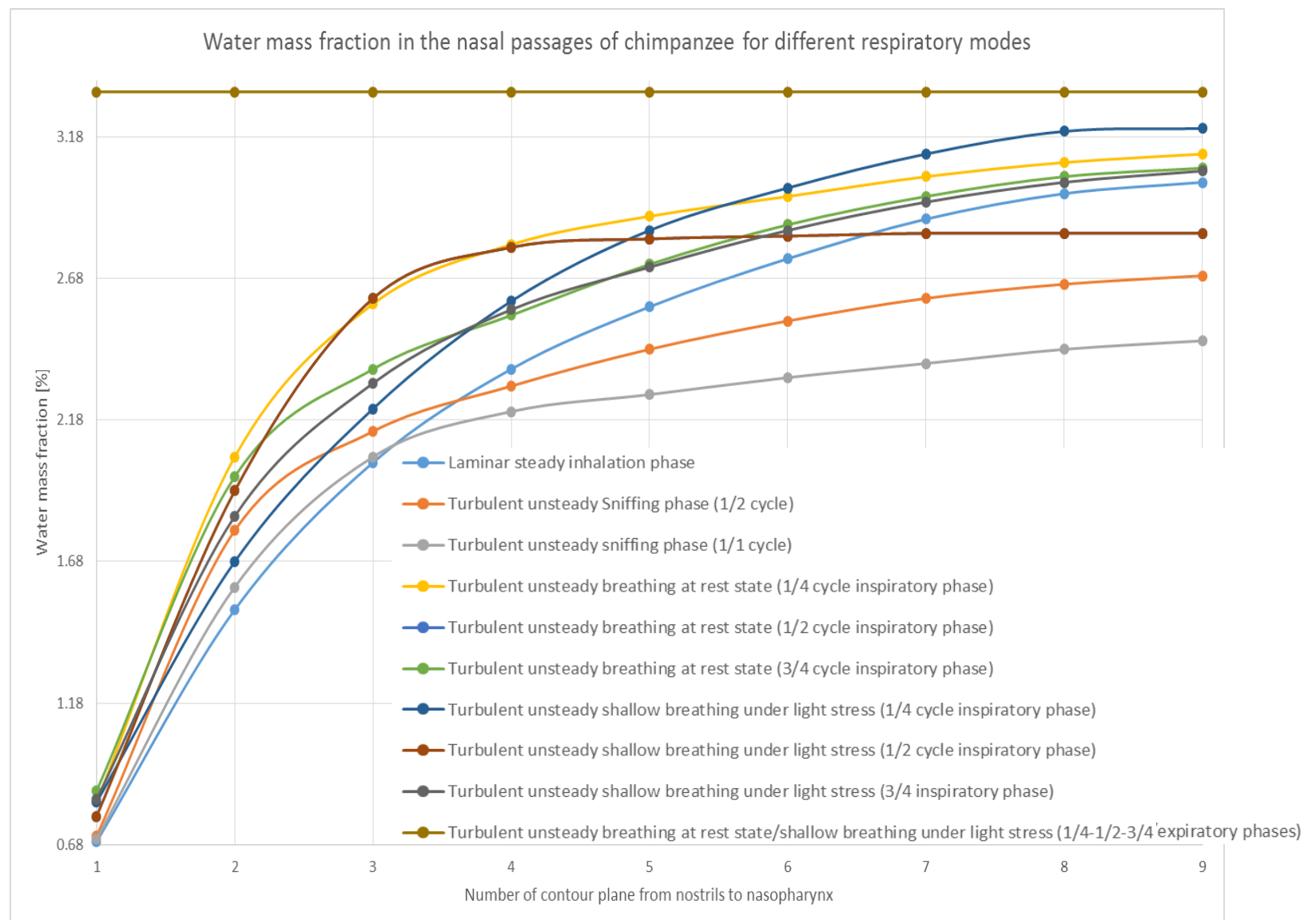

Figure 6. Water mass fraction distribution within chimpanzee nasal cavity for different breathing modes: Horizontal axis represents number of contour plane from nostrils to pharynx. Vertical axis represents water mass fraction [\%].

We conclude from the above graphs that assuming an inhalation laminar steady state to investigate chimpanzee nasal air-conditioning principles, in order to compare nasal cavities performance of human, chimp, and macaque [12], may be exaggerated.

Acknowledgments: The authors thank T. Nishimura for providing the CT scans used in this study, K. Kumahata for providing the original source code of CFD method, and F. Mori for his technical support.
Conflicting Interests: The authors have declared that no conflicting interests exist.

\section{References}

1. P. Cole, "Modification of inspired air. In The Nose: Upper Airway Physiology and the Atmospheric Environment," pp. 350-375, 1982.

2. D. Elad, M. Wolf, T. Keck, "Air-conditioning in the human nasal cavity," Respir. Physiol. Neurobiol., pp. 163, 121-127, 2008.

3. J. Lindemann, R. Leiacker, G. Rettinger, T. Keck, "The relationship between water vapour saturation of inhaled air and nasal patency," European Respiratory Journal, 21, pp. 313-316, 2003. 
4. J. S. Kimbell, R. P. Subramaniam, E. A. Gross, P. M. Schlosser, K. T. Morgan, "Dosimetry Modeling of Inhaled Formaldehyde: Comparisons of Local Flux Predictions in the Rat, Monkey, and Human Nasal Passages," Toxicological Sciences, 64(1), pp. 100-110, 2001.

5. R. A. Corley, K. R. Minard, S. Kabilan, D. R. Einstein, A. P. Kuprat, J. R. Harkema, J. S. Kimbell, M. L. Gargas, J. H. Kinzell, "Magnetic resonance imaging and computational fluid dynamics (CFD) simulations of rabbit nasal airflows for the development of hybrid CFD/PBPK models,". Inhalation Toxicology vol. 21, Iss. 6, 2009.

6. W. Zehong, X. Zhixiang, L. Bo, X. Fuqiang, "Numerical simulation of airway dimension effects on airflow patterns and odorant deposition patterns in the rat nasal cavity," PLOS ONE 8 (10), 2013.

7. P. A. Green, B. V. Valkenburgh, B. Pang, D. Bird, T. Rowe, A. Curtis, "Respiratory and olfactory turbinal size in canid and arctoid carnivorans," $J$ Anat., 221, pp. 609-621, 2012.

8. M. J. Lawson, B. A. Craven, E. G. Paterson, G. S. Settles, "A computational study of odorant transport and deposition in the canine nasal cavity: implications for olfaction," Chem Senses, 37 (6), pp. 553-566, 2012.

9. J. M. Bourke, WM. Ruger Porter, R. C. Ridgely, T. R. Lyson, E. R. Schachner, P. R. Bell, L. M. Witmer, "Breathing Life into Dinosaurs: Tackling Challenges of Soft-Tissue Restoration and Nasal Airflow in Extinct Species," Anat. Rec., 297, pp. 2148-2186, 2014.

10. A. N. Ranslow, J. P. Richter, T. Neuberger, B. V. Valkenburgh, C. R. Rumple, A. P. Quigley, B. Pang, M. H. Krane, B. A. Craven, "Reconstruction and Morphometric Analysis of the Nasal Airway of the White-Tailed Deer (Odocoileus virginianus) and Implications Regarding Respiratory and Olfactory Airflow," The Anatomical Record, 297, pp. 2138-2147, 2014.

11. F. Mori, S. Hanida, K. Kumahata, T. M. Nishiwaki, J. Suzuki, T. Matsuzawa, "Minor contributions of the maxillary sinus to the air-conditioning performance in macaque monkeys," J Exp Biol., 218, pp. 2394-2401, 2015.

12. T. Nishimura, F. Mori, S. Hanida, K. Kumahata, S. Ishikawa, "Impaired Air Conditioning within the Nasal Cavity in Flat-Faced Homo," PLoS Comput Biol, 12(3), 2016.

13. D. Simmen, J. L. Scherrer, K. Moe, B. Heinz, "A Dynamic and Direct Visualization Model for the Study of Nasal Airflow," Arch Otolaryngol Head Neck Surg., 125(9), pp. 1015-1021, 1999.
14. X. B. Chen, H. P. Lee, V. F. Hin Chong, D. Y. Wang, "Assessment of septal deviation effects on nasal air flow: A computational fluid dynamics model," The Laryngoscope, 119, pp. 1730-1736, 2009.

15. K. Samarat, T. Matsuzawa, "A Computational Model of the Anatomy of Realistic Chimpanzee Nasal Airways", IJIR, 2 (2), pp. 357-364, 2016.

16. T. Matsuzawa, M. Tomonaga, M.Tanaka, "Cognitive development in chimpanzees," 2006.

17. K. Kumahata, F. Mori, S. Ishikawa, T. Matsuzawa, "Nasal flow simulation using heat and humidity models," J Biomech Sci Engineer, 5, pp. 565-577, 2010.

18. K. Samarat, K. Kumahata, S. Hanida, T. Nishimura, F. Mori, S. Ishikawa, T. Matsuzawa, "Application of computational fluid dynamics to simulate a steady airflow in all regions of chimpanzee nasal cavity," Procedia Engineering, 61C, pp. 264-269, 2013.

19. Worthington, J., Young, I. S. and Altringham, J. D.: "The relationship between body-mass and ventilation rate in mammals", J. Exp. Biol. 161, pp.533-536 (1991).

20. Stahl, W. R.: "Scaling of respiratory variables in mammals," J. Appl. Physiol. 22, pp. 453-460 (1967).

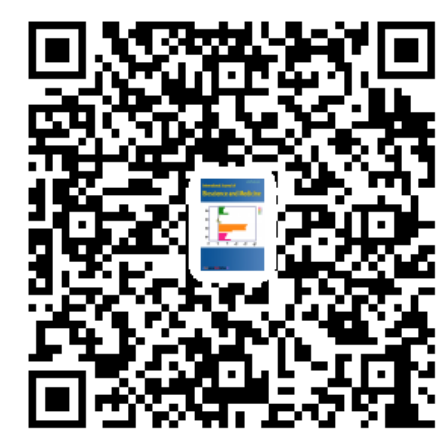

